Relations industrielles

Industrial Relations

\title{
CETA: Manpower Programs Under Local Control, by William Mirengoff and Lester Rindler, Washington, National Academy of Sciences, 1978, 327 pp.
}

\section{Jean Sexton}

Volume 34, numéro 4, 1979

URI : https://id.erudit.org/iderudit/029019ar

DOI : https://doi.org/10.7202/029019ar

Aller au sommaire du numéro

Éditeur(s)

Département des relations industrielles de l'Université Laval

ISSN

0034-379X (imprimé)

1703-8138 (numérique)

Découvrir la revue

Citer ce compte rendu

Sexton, J. (1979). Compte rendu de [CETA: Manpower Programs Under Local Control, by William Mirengoff and Lester Rindler, Washington, National Academy of Sciences, 1978, 327 pp.] Relations industrielles / Industrial Relations, 34(4), 824-825. https://doi.org/10.7202/029019ar

Tous droits réservés (C) Département des relations industrielles de l'Université Laval, 1979
Ce document est protégé par la loi sur le droit d'auteur. L’utilisation des services d'Érudit (y compris la reproduction) est assujettie à sa politique d'utilisation que vous pouvez consulter en ligne.

https://apropos.erudit.org/fr/usagers/politique-dutilisation/ 


\section{RECENSIONS}

\section{BOOK REVIEWS}

CETA: Manpower Programs Under Local Control, by William Mirengoff and Lester Rindler, Washington, National Academy of Sciences, 1978, 327 pp.

En décembre 1973, le gouvernement américain abrogeait sa principale loi de maind'oeuvre, alors vieille de onze ans, le Manpower Development and Training Act (MDTA) adopté dans la foulée du "automation mania" du début des années "60 et le remplaçait par le Comprehensive Employment and Training Act (CETA), une nouvelle loi de main-d'oeuvre qui adoptait une nouvelle approche qui surprend encore celui qui identifie Etats-Unis et conservatisme libéraliste à outrance. En effet, parmi ses principales caractéristiques, ce nouveau CETA, non seulement consacrait une nouvelle approche en décentralisant les services de main-d'oeuvre principalement par l'application du concept de "prime sponsor", mais regroupait, pour plusieurs de façon surprenante, ses principales interventions en matière de main-d'oeuvre sous un même chapeau, sous une même loi, faisant ainsi en quelque sorte un pas important vers une véritable loi intégrée des services de maind'oeuvre aux Etats-Unis.

L'expérience américaine en matière de main-d'oeuvre a toujours insisté, depuis le MDTA, sur l'aspect évaluation des programmes de main-d'oeuvre financés par les fonds publics et est même allée jusqu'à expressément l'exiger par la loi elle-même. Cette tradition a été gardée et même amplifiée par le CETA où non seulement le législateur améri- cain prévoit et exige que des évaluations soient faites de programmes de maind'oeuvre, mais en prévoit même la méthodologie.

On doit donc comprendre que cet aspect évaluation est non seulement fortement ancré dans l'approche américaine de maind'oeuvre, mais également qu'il a été appliqué de façon rigoureuse, comme en témoignent d'ailleurs les compte-rendus des différents comités du gouvernement américain.

C'est dans ce contexte, que le National Research Council établissait en 1974 le Committee on Evaluation of Employment and Training Programs.

Le présent volume, le rapport final de ce Comité, étudie dans un premier tennps les différences entre les programmes prévus au Titre I du CETA avec leurs prédécesseurs et compare les objectifs du législateur avec les résultats obtenus. En second lieu, ce livre étudie l'impact des programmes d'emploi dans le service public sur les programmes d'orientation structurelle du titre I du CETA et le degré d'atteinte de l'objectif principal de ce titre VI à savoir la création de nouveaux emplois. Finalement, le Comité publie un certain nombre de recommandations.

Après avoir présenté le CETA et ses objectifs (ch. 1), cet ouvrage examine successivement les sujets suivants: les ressources des programmes (ch. 2), la planification de maind'oeuvre depuis l'expérience des CAMPS (ch. 3), l'aspect administratif des programmes (ch. 4), les choix de programmes (ch. 5), la 
livraison des services du Titre I (ch. 6), la création d'emploi dans le service public (ch. 7), la clientèle (ch. 8) et l'efficacité des programmes (ch. 8). Le chapitre 9, pour sa part, est entièrement consacré aux conclusions et recommandations.

Cet ouvrage est écrit dans un langage simple et accessible, et sa présentation claire en facilite la lecture tant par des tableaux bien faits que par les résumés succints a la fin de chacun des chapitres.

En conclusion, celui qui veut s'intéresser a l'expérience récente des Etats-Unis en matière de main-d'oeuvre trouvera dans cet ouvrage un recueil de renseignements très utiles qui va surement l'inciter à pousser son étude plus loin.

\section{Jean SEXTON}

Université Laval

The Current Industrial Relations Scene in Canada 1979, by W.D. Wood and Pradeep Kumar (Ed.), Kingston, Industrial Relations Centre, Queen's University, 1979, $695 \mathrm{pp}$.

Canadian Industrial Relations Information, Sources, Technical Notes and Glossary, by Pradeep Kumar, Kingston, Industrial Relations Centre, Queen's University, 1979, 166 pp.

Le Centre des relations industrielles de l'Université Queen vient de publier son précieux annuaire de 1979 comprenant la plupart des informations touchant les relations du travail au Canada. Cette édition présente les mêmes grandes rubriques: économie, maind'oeuvre et marché du travail, législation du travail et politique sociale, syndicalisme, négociation collective, salaires, productivité et couts de la main-d'oeuvre, documentation. Toutes les données ont été mises à jour et on a indiqué les changements intervenus depuis l'année précédente.

Les auteurs ont innové en publiant dans un ouvrage distinct, Canadian Industrial Relations Information, les sources d'infor- mation, les explications techniques ainsi qu'un glossaire des termes couramment utilisés en relations industrielles. Cet ouvrage, qui a une valeur permanente, est particulièrement utile pour connaître non seulement les sources des informations, mais encore pour savoir comment les données sont recueillies et la portée de l'interprétation qu'on peut leur attribuer.

Les deux ouvrages peuvent être achetés séparément, mais un prix spécial est accordé à ceux qui se les procurent ensemble.

Le Centre des relations industrielles de Queen, en publiant chaque année cette compilation d'informations, rend un service inappréciable à tous ceux qui s'occupent des relations du travail. Avec la disparition de La Gazette du travail et de l'annuaire Organisation des travailleurs au Canada, ces ouvrages sont davantage indispensables. Il faut espérer que le Centre continuera.

\section{Gérard DION}

Université Laval

International Labour Law Reports, par Zvi H. Bar-Niv (Chairman), Benjamin Aaron, Thilo Ramm, Folke Schmidt, Jean Maurice Verdier, Kenneth William Wedderburn (Editorial Board), Peter Elman (Coordinating Editor) Alphen aan den Rijn (Pays Bas), Sijthoff \& Noordhoff, 1978, volume 1, $383 \mathrm{pp}$.

Voici le premier tome des International Labour Law Reports qui inaugure une série de publications annuelles de jugements de 10 différentes juridictions compétentes dans le domaine du travail en Europe, en Israël, au Japon et aux Etats-Unis.

Il s'agit là d'un choix destiné à offrir aux lecteurs visés - magistrats, praticiens du droit du travail, spécialistes de relations industrielles, enseignants et étudiants -, des décisions reflétant des approches et des systèmes légaux variés dans un certain nombre de pays industrialisés ayant comme dénominateur commun la libre négociation collective. 